\title{
Neutrophil to lymphocyte and platelet to lymphocyte ratios as biomarkers to predict relapse and survival in posthepatectomy HBV-related hepatocellular carcinoma: a meta-analysis and preliminary immune perspective
}

\author{
Xia Zheng ${ }^{1}$, Bin Ye ${ }^{2}$, Yudong Gou ${ }^{1}$, Zixiong Li $^{3}$, Chao Chen ${ }^{3}$, Feng Liao ${ }^{3}$, Xiufeng Liu ${ }^{3}$, Shukui Qin ${ }^{1,3}$ \\ ${ }^{1}$ Nanjing University of Chinese Medicine, Nanjing, China; ${ }^{2}$ Shenogene Pharma Group, Beijing, China; ${ }^{3}$ Department of Oncology, Nanjing Jinling \\ Hospital, Nanjing, China \\ Contributions: (I) Conception and design: S Qin; (II) Administrative support: X Liu, F Liao; (III) Provision of study materials or patients: X Zheng, Y \\ Gou; (IV) Collection and assembly of data: Z Li, C Chen; (V) Data analysis and interpretation: B Ye; (VI) Manuscript writing: All authors; (VII) Final \\ approval of manuscript: All authors. \\ Correspondence to: Shukui Qin. Yanggongjing NO. 34-34, Taiping South Road, Nanjing, China. Email: qinsk@csco.org.cn.
}

Background: Hepatitis B virus (HBV) infection represents the major etiology of hepatocellular carcinoma (HCC) and results in poor outcomes. Accumulating evidence suggests that composite immune cell-based biomarkers such as neutrophil to lymphocyte ratio (NLR) and platelet to lymphocyte ratio (PLR) have prognostic value in postoperative HCC patients. However, due to the complexity, differential etiology, and the presence of variable confounding factors in different studies, the relationship between these markers with clinical outcomes in HBV-related posthepatectomy HCC is unclear from an immune perspective. Thus, this meta-analysis was conducted to determine NLR and PLR and assess their relation to overall survival (OS) and recurrence-free survival (RFS) in patients with post-hepatectomy HCC with HBV infection.

Methods: The databases PubMed, Embase, Scopus, and Cochrane Library were searched using relevant keywords. We included studies which compared the outcomes of RFS and OS between different levels of NLR and PLR in HBV-related HCC patients who had undergone hepatectomy. Hazard ratios (HRs) and their $95 \%$ confidence intervals (CIs) were considered as effective measures and were calculated by a pooled analysis. Evidence supporting the association of neutrophils, platelets, and lymphocytes with HBV infection, liver injury, and tumor progression in HCC was evaluated.

Results: A total of 11 cohort studies with 5083 patients were included. Elevated NLR was significantly associated with poor RFS (HR: $1.28,95 \% \mathrm{CI}: 1.09-1.50, \mathrm{P}=0.000)$ and poor OS (HR: $1.64,95 \% \mathrm{CI}$ : 1.32-2.03, $\mathrm{P}=0.000)$. Decreased PLR was significantly associated with a low risk of posthepatectomy relapse (HR: 1.40, 95\% CI: 1.28-1.53, P=0.000) and better survival (HR: 1.63, 95\% CI: $1.42-1.87, \mathrm{P}=0.000$ ). The subgroup and sensitivity analysis showed consistent and stable results.

Conclusions: Both NLR and PLR can be used as biomarkers for the prediction of RFS and OS in patients with HBV-associated HCC after hepatectomy.

Keywords: Neutrophil to lymphocyte ratio (NLR); platelet to lymphocyte ratio (PLR); hepatectomy; Hepatitis B virus (HBV)-related hepatocellular carcinoma (HCC); meta-analysis

Submitted Oct 22, 2020. Accepted for publication Jan 29, 2021.

doi: $10.21037 /$ tcr-20-3125

View this article at: http://dx.doi.org/10.21037/tcr-20-3125 


\section{Introduction}

Hepatocellular carcinoma (HCC) represents the sixth most common malignancy and the third leading cause of cancerrelated deaths globally (1). Due to the lack of specific symptoms at early stages, most patients are diagnosed at an advanced stage. This situation severely limits treatment options-sometimes to only palliative therapy-and has led to poor overall survival (OS). However, most early stage HCC patients could be cured if they have adequate liver function. Liver resection, liver transplantation, and radiofrequency ablation (RFA) are the main curative strategies for this disease. Hepatectomy, often the most preferred treatment option, is widely offered to early stage and some middle-stage HCC patients. However, because of the high risk of progression and lack of satisfactory adjuvant therapies (2), up to $70 \%$ of hepatectomy patients suffer from cancer relapse within 5 years, resulting in poor prognosis ${ }^{[1]}$. Therefore, it is critical to identify potential biomarkers associated with recurrence and mortality risk posthepatectomy. Currently, clinicopathologic characteristics such as tumor size, vascular invasion, and margin involvement are often used to predict recurrence and survival outcomes (3).

Cancer-associated infections, especially viral infections that often cause chronic inflammation, are responsible for more than $15 \%$ of cancer cases worldwide (4). Chronic inflammatory conditions such as hepatitis contribute significantly to tumorigenesis, cancer progression, treatment failure, and recurrence. Unlike in Western countries, in Asian countries, hepatitis B virus (HBV) infection accounts for up to $85 \%$ of new cases and is the leading cause of HCC development (5). Therefore, HBV-related HCC biology and chronic inflammatory conditions directly impact the clinical outcomes of treatments such as liver resection and transplantation. A recently published, multicenter, retrospective study showed that viral status along with other factors such as tumor size, tumor differentiation, margin status, vascular invasion, and Child-Pugh score significantly contributed to long-term survival in hepatectomy patients with HBV-related HCC (6).

Neutrophil to lymphocyte ratio (NLR) and platelet to lymphocyte ratio (PLR) are widely accepted inflammatory and immune biomarkers that often reflect cancer immune status (7). Previous studies have demonstrated that these markers could predict cancer recurrence and patient survival in postoperative gastric cancer (8), breast cancer (9), colorectal cancer (10), and HCC (11). However, contradictions or inconsistencies have resulted from differences in study design and limited sample sizes. Notably, HCC often has considerably high heterogeneity and multiple etiologies, such as hepatitis $\mathrm{B} / \mathrm{C}$ viral infection and alcohol abuse. Infection with $\mathrm{HBV}$ is the most common etiology in the Asian population and in high-mortality HCC patients. Therefore, the prognostic value of these biomarkers for HCC associated with HBV status is of great interest. Previous studies have revealed that increased NLR and PLR are associated with poor outcomes for posthepatectomy HBV-related HCC patients $(12,13)$. However, according to studies reported by Li et al. (14) and Rungsakulkij et al. (15), there are no obvious connections between patient outcomes and NLR or PLR levels.

In addition to collecting and screening previously published data, we re-explored the predictive values of both NLR and PLR in hepatectomy HCC patients with HBV infection. The potential regulatory functions of immune cells of NLR and PLR including neutrophils, lymphocytes, and platelets in $\mathrm{HBV}$ infection and $\mathrm{HCC}$ tumor progression were characterized. We present the following article in accordance with the Preferred Reporting Items for Systematic Reviews and Meta-Analyses (PRISMA) reporting checklist (available at http://dx.doi.org/10.21037/ tcr-20-3125).

\section{Methods}

\section{Search strategy and criteria}

Published studies potentially related to HCC hepatectomy and NLR and PLR were extracted from the PubMed, Embase, Scopus, and Cochrane Library databases in December 2020. The key words "hepatocellular carcinoma", "neutrophil lymphocyte ratio", "platelet lymphocyte ratio", and "hepatectomy" and related abbreviations were applied for screening and identification of candidate studies to be included in the meta-analysis. Multiple synonyms were also utilized. Eligible studies were assessed using the following criteria: (I) all post-hepatectomy patients had been diagnosed with HCC by pathological examination; (II) the association between NLR and RFS/OS and between PLR and RFS/OS were evaluated; (III) the peripheral blood samples were obtained before surgery. Exclusion criteria for this meta-analysis were as follows: (I) the study was not restricted to humans; (II) the published materials were review articles, case reports, or conference abstracts; (III) studies had insufficient data to calculate a hazard ratio (HR) 


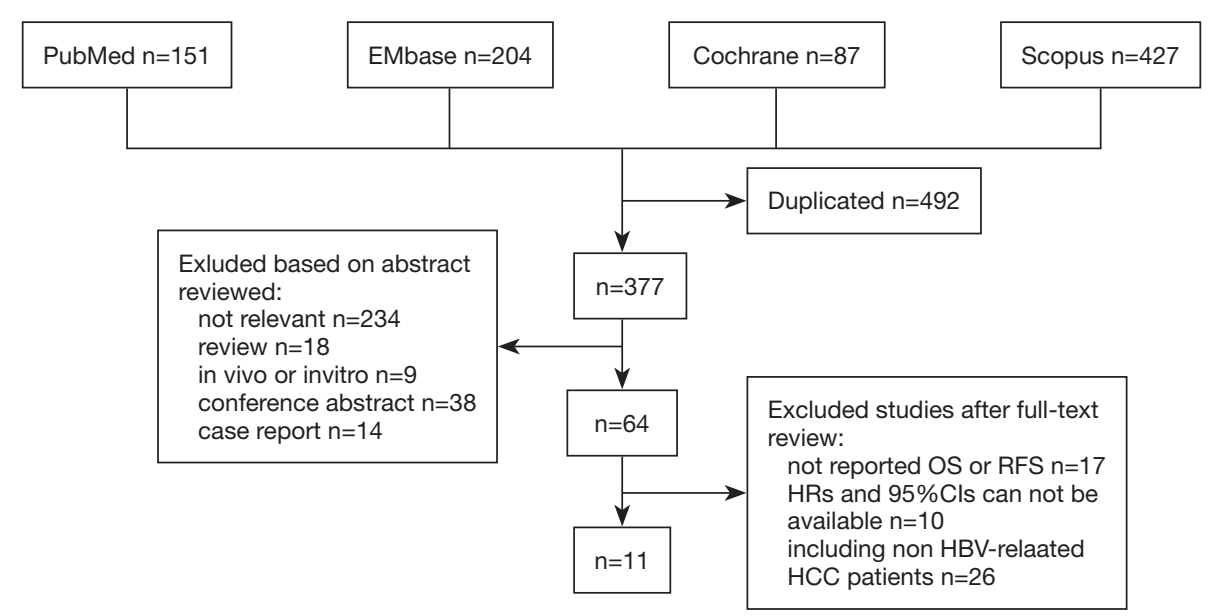

Figure 1 Flow diagram of study selection.

and $95 \%$ confidence interval (CI); (VI) studies included non HVB-related HCC patients. If multiple studies were reported by the same team from the same institute or were performed at the same time, only the most recent article was included.

\section{Date management and outcome assessment}

Available articles were independently selected and reviewed by 2 authors through abstract and full-text reading. In the case of disagreement between them, final decisions were made by a senior author. The HRs and $95 \%$ CIs of OS and recurrence-free survival (RFS) were collected and recognized as the effective measurements. We preferentially extracted the HRs and their 95\% CIs calculated by multivariate analysis to achieve better accuracy.

\section{Quality assessment}

The UK Cochrane Centre of Evidence (2009) (16) was used to estimate the evidence level of the studies. The quality of the retrospective cohort studies was assessed using the NewcastleOttawa Scale (16). This scale consists of three factors: the selection of patients, comparability of the study groups, and assessment of outcome. The maximum total score was 9; studies with scores $\geq 6$ were defined as high-quality studies, and this was a presetting selection criterion in this report.

\section{Statistical analysis}

The HRs and associated $95 \%$ CIs were calculated to pool the functional outcomes. Statistical heterogeneity among the studies was assessed using chi-square tests with the significance set to $\mathrm{P}<0.05$ or $\mathrm{I}^{2}>50 \%$. A fixed-effects model was utilized if there was no evident heterogeneity; otherwise, we selected a random-effects model to minimize the heterogeneity followed with subgroup and sensitivity analysis. Funnel plots, Egger's test, and Begg's test were used to examine publication bias. All statistical analyses were performed using STATA version 14.0 (Stata statistical software, College Station, TX, USA).

\section{Results}

\section{Characteristics of the selected articles}

In total, 869 articles were identified from the 4 online databases, and 492 were removed due to duplication. After scanning the titles and abstracts, 313 articles were excluded; of these, 234 were unrelated, 18 were review papers, 14 were case reports, 38 were conference abstracts, and 9 were experimental studies. According to the above exclusion criteria, 11 eligible studies were identified and included in our meta-analysis following confirmation by reviewing the full text (Figure 1). The most common reason for exclusion was that the studies included non-HBV-related cases $(\mathrm{n}=26)$. A total of 17 studies were excluded because they did not report the OS or RFS.

Overall, 5083 patients from the 11 retrospective cohort studies were included in this study $(12-15,17-23)$. The level of evidence was $2 \mathrm{a}$. There were 10 and 8 studies reporting the prognostic role of NLR and PLR, respectively, and 6 reported both. The cutoff values of NLR and PLR 
Table 1 characteristics of included studies

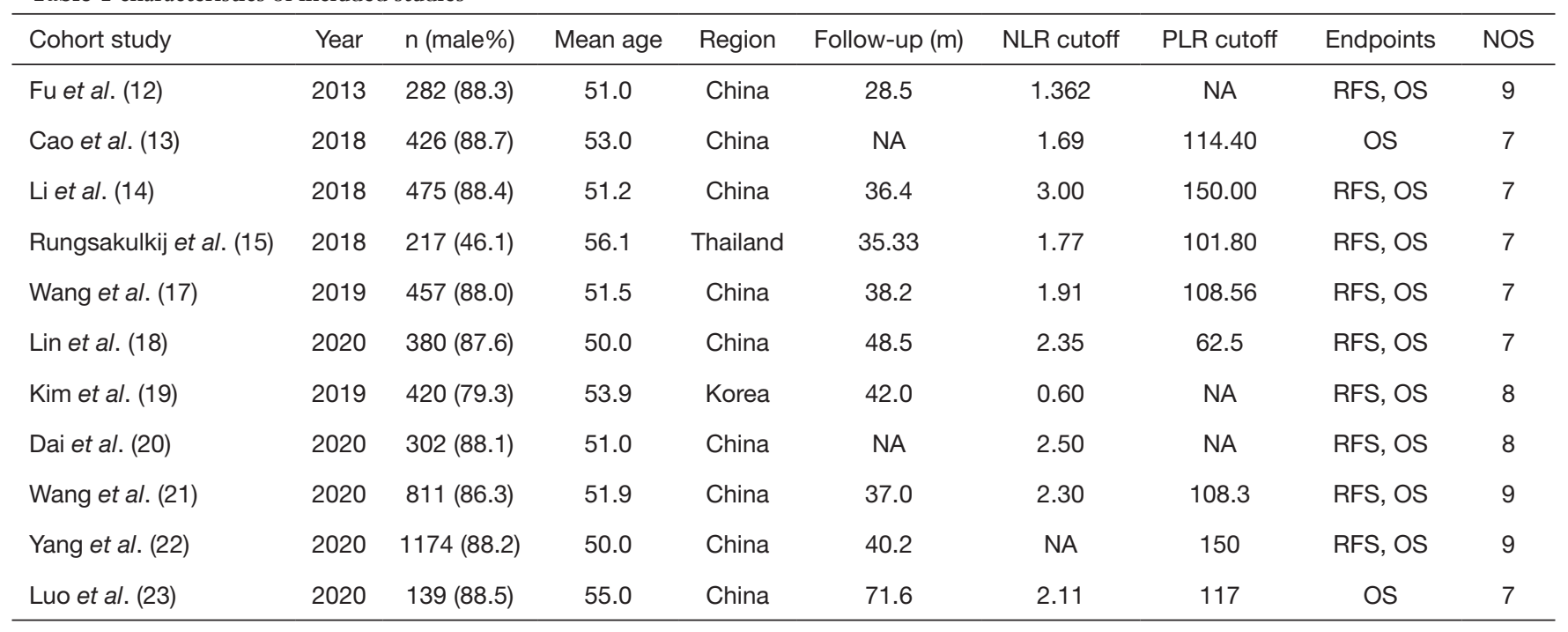

NLR, neutrophil to lymphocyte ratio; PLR, platelet to lymphocyte ratio; RFS, recurrence free survival; OS, overall survival.

were $0.6-3.0$ and $62.5-150.0$, respectively. Based on the Newcastle-Ottawa Scale, all studies received a quality score of 6-9 (Table 1).

\section{NLR for prediction of posthepatectomy survival}

The NLR predicting RFS of HCC patients after surgery was reported in a total of 8 cohorts with 3,344 patients. According to the heterogeneity $\left(\mathrm{I}^{2}=57.3 \%, \mathrm{P}=0.022\right)$, a random-effects model was used to analyze these data, and we found that an elevated NLR significantly correlated with poor RFS (HR: 1.28, 95\% CI: 1.09-1.50, $\mathrm{P}=0.000$ ) (Table 2, Figure 2A). Similarly, there was a significant correlation between an elevated NLR and a poor OS in HBV-related HCC patients who underwent hepatectomy (HR: 1.64, 95\% CI: 1.32-2.03, $\mathrm{P}=0.000$ ) (Table 2, Figure $2 B$ ) according to the pooled analysis of the 10 cohorts with the random-effects model $\left(\mathrm{I}^{2}=67.2 \%\right.$, $\mathrm{P}=0.004)$. Thus, a decreased NLR predicted a better RFS and OS in posthepatectomy HCC patients with HBV infection.

\section{PLR for prediction of posthepatectomy survival}

A pooled analysis of the 6 cohorts with a fixed-effect model $\left(\mathrm{I}^{2}=44.0 \%, \mathrm{P}=0.106\right)$ showed that an increased PLR was significantly associated with the high risk of posthepatectomy relapse (HR: 1.40, 95\% CI: 1.28-1.53, P=0.000; Table 2, Figure 2C). Furthermore, analysis of the 6 cohorts with $3051 \mathrm{HBV}$-infected HCC patients showed that low PLR was significantly correlated with better OS (HR: 1.63, 95\%
CI: 1.42-1.87, P=0.000; Table 2, Figure 2D). These findings indicated that a lower PLR was linked to better outcomes in HBV-related HCC patients after hepatectomy.

\section{Subgroup analysis of the prognostic value of NLR}

Due to obvious heterogeneity among the studies related to the prognostic role of NLR in HBV-related HCC patients, we performed a subgroup analysis by area, analysis methods, and NLR cutoff value. This revealed that elevated NLR was associated with poor RFS in studies performed in China (HR: $1.38,95 \%$ CI: $1.16-1.63, \mathrm{P}=0.000)$. In addition, we found that increased NLR was an obvious risk factor for tumor recurrence when pooled analyzed the effective measures which calculated with multivariate analysis (HR: 1.58, 95\% CI: 1.16-2.12, $\mathrm{P}=0.002)$. In the subgroup analysis of cutoff values, a decreased NLR was associated with better RFS in the studies with cutoff values $>2$ (HR: $1.41,95 \%$ CI: 1.06-1.88, $\mathrm{P}=0.034$; Table 3).

The other subgroup analysis was performed on those studies examining NLR prediction of mortality risk. NLR was significantly associated with poor OS in the studies from China (HR: 1.79, 95\% CI: 1.53-2.10, $\mathrm{P}=0.000$ ). The survival prognostic value of NLR was significant, independent of the analysis method or cutoff value (Table 4).

\section{Sensitivity and publication bias analysis}

We performed a sensitivity analysis with the leave-oneout method to examine the stability of the pooled analysis 
Table 2 Result of meta-analysis of interested outcomes

\begin{tabular}{|c|c|c|c|c|c|c|c|c|}
\hline Outcomes & Cohort number & Case number & HR (95\% Cl)-Model & $\mathrm{P}$ & \multicolumn{2}{|c|}{ Heterogeneity } & \multicolumn{2}{|c|}{ Publication bias } \\
\hline \multicolumn{9}{|l|}{ RFS } \\
\hline NLR & 8 & 3,344 & 1.28 (1.09-1.50)-random & 0.000 & 57.3 & 0.022 & 0.222 & 0.710 \\
\hline PLR & 6 & 3,515 & 1.40 (1.28-1.53)-fixed & 0.000 & 44.9 & 0.106 & 0.099 & 0.133 \\
\hline \multicolumn{9}{|l|}{ OS } \\
\hline NLR & 10 & 3,909 & 1.64 (1.32-2.03)-random & 0.000 & 62.7 & 0.004 & 0.798 & 0.592 \\
\hline PLR & 6 & 3,051 & 1.63 (1.42-1.87)-fixed & 0.000 & 41.7 & 0.127 & 0.950 & 1.000 \\
\hline
\end{tabular}

NLR, neutrophil to lymphocyte ratio; PLR, platelet to lymphocyte ratio; RFS, recurrence free survival; OS, overall survival.

A Study ID

Fu et al (2013) Li et al (2018) Rungsakulkij at al (2018) Wang et al (2019) Lin et al (2019) Kim et al (2019) Dai et al (2020) Wang et al (2020) Overall (I-squared $=57.3 \%, p=0.022$ ) $\frac{\text { NOTE: Weighs are trom random effects anaysis }}{.251}$

C Study ID

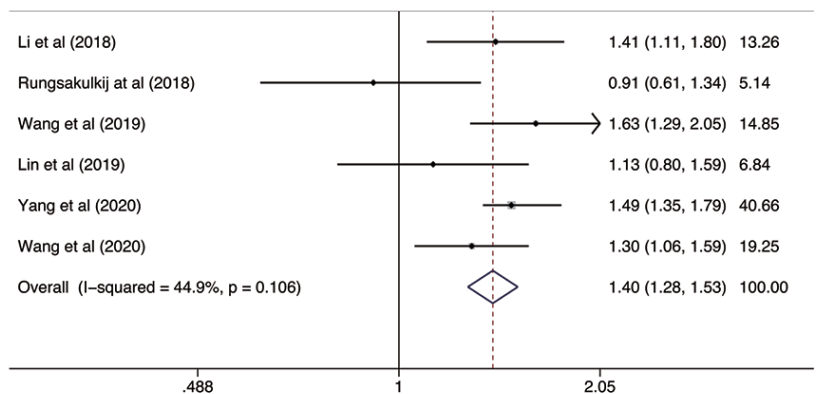

B Study ID HR $(95 \% \mathrm{Cl}) \quad$ Weight

\begin{tabular}{|c|c|c|}
\hline Fu et al (2013) & $\rightarrow$ & $1.43(1.04,1.97) 12.63$ \\
\hline Cao et al (2017) & $\longrightarrow$ & $2.62(1.78,3.85) 11.14$ \\
\hline Li et al (2018) & & $1.35(0.93,1.97) 11.35$ \\
\hline Rungsakulkij at al (2018) & 5 & $1.07(0.82,1.39) 13.83$ \\
\hline Wang et al (2019) & & $1.71(1.27,2.30) 13.09$ \\
\hline Lin et al (2019) & & $1.80(1.20,2.70) 10.77$ \\
\hline Kim et al (2019) & & $0.36(0.08,1.55) 1.91$ \\
\hline Dai et al (2020) & & $1.91(1.08,3.38) 7.75$ \\
\hline Wang et al (2020) & & $2.15(1.45,3.18) 10.99$ \\
\hline Luo et al (2020) & & $2.11(1.09,4.08) 6.54$ \\
\hline Overall (I-squared $=62.7 \%, p=0.004$ ) & & $1.64(1.32,2.03) 100.00$ \\
\hline NOTE: Woights are trom random effocts analyaits & & \\
\hline
\end{tabular}

$\mathrm{D}$ Study ID

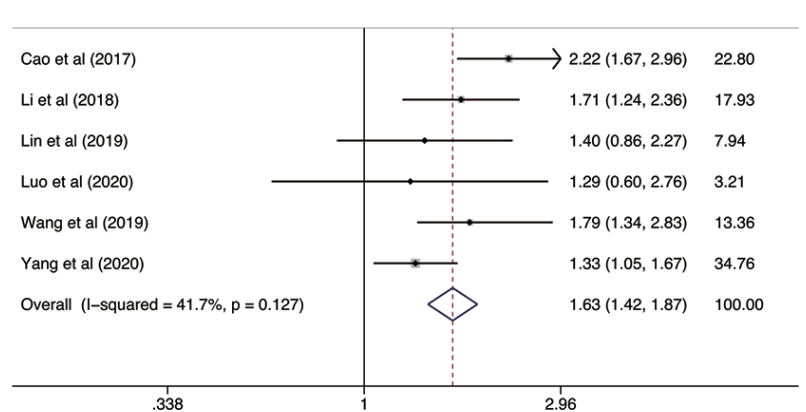

Figure 2 Forest plots of the correlation between NLR and PLR in predicting HBV-related HCC patients outcomes. (A) NLR predicts recurrence risk. (B) NLR predicts survival. (C) PLR predicts relapse risk. (D) PLR predicts mortality risk. NLR, neutrophil to lymphocyte ratio; PLR, platelet to lymphocyte ratio; HBV, hepatitis B virus; HCC, hepatocellular carcinoma. 
Table 3 Subgroup pooled analysis of the studies related to NLR and tumor recurrence risk

\begin{tabular}{|c|c|c|c|c|c|c|}
\hline Subgroups & Cohort number & Case number & HR (95\%Cl) & $\mathrm{P}$ & \multicolumn{2}{|c|}{ Heterogeneity } \\
\hline \multicolumn{7}{|l|}{ Area } \\
\hline China & 6 & 2,707 & 1.38 (1.16-1.63) & 0.000 & 43.7 & 0.114 \\
\hline Non-China & 2 & 637 & $1.04(0.89-1.21)$ & 0.651 & 0.0 & 0.514 \\
\hline Univariate & 5 & 2,194 & $1.14(0.99-1.30)$ & 0.063 & 18.1 & 0.299 \\
\hline Multivariate & 3 & 1,150 & $1.58(1.16-2.12)$ & 0.002 & 57.5 & 0.095 \\
\hline \multicolumn{7}{|l|}{ Cut-off } \\
\hline$\leq 2$ & 4 & 1,569 & $1.20(0.98-1.45)$ & 0.106 & 51.0 & 0.106 \\
\hline
\end{tabular}

NLR, neutrophil to lymphocyte ratio.

Table 4 Subgroup pooled analysis of the studies related to NLR and survival

\begin{tabular}{|c|c|c|c|c|c|c|}
\hline Subgroups & Cohort number & Case number & HR (95\% Cl) & $\mathrm{P}$ & \multicolumn{2}{|c|}{ Heterogeneity } \\
\hline \multicolumn{7}{|l|}{ Area } \\
\hline China & 8 & 3,272 & $1.79(1.53-2.10)$ & 0.000 & 21.8 & 0.256 \\
\hline Non-China & 2 & 637 & $0.78(0.31-2.05)$ & 0.635 & 51.7 & 0.150 \\
\hline Univariate & 6 & 1,933 & $1.43(1.06-1.92)$ & 0.018 & 55.6 & 0.046 \\
\hline Multivariate & 4 & 1,976 & $1.89(1.47-2.43)$ & 0.000 & 53.3 & 0.092 \\
\hline \multicolumn{7}{|l|}{ Cut-off } \\
\hline$\leq 2$ & 5 & 1,082 & $1.48(1.02-2.13)$ & 0.037 & 78.7 & 0.001 \\
\hline
\end{tabular}

NLR, neutrophil to lymphocyte ratio.

results about NLR predictive value. The statistical significance of the results did not change when any single study was excluded, which indicated the results of this metaanalysis were stable and robust (Figure 3).

No evidence of obvious publication bias was detected according to the results of Egger's test $(\mathrm{P}>0.05)$, Begg's test $(\mathrm{P}>0.05)$ (Table 2$)$, and the symmetry of funnel plots (Figure 4).

\section{Immune cells of NLR, PLR, and functions in HBV-related} HCC

Neutrophils and platelets are involved in the suppression of immune functions of CD8+ T cells and promote tumor progression and early angiogenesis by recruiting macrophages and regulatory $\mathrm{T}$ cells (Tregs) (24-32) (Table 5). Therefore, high neutrophil and platelet counts would directly contribute to high NLR and PLR. However, different lymphocyte 

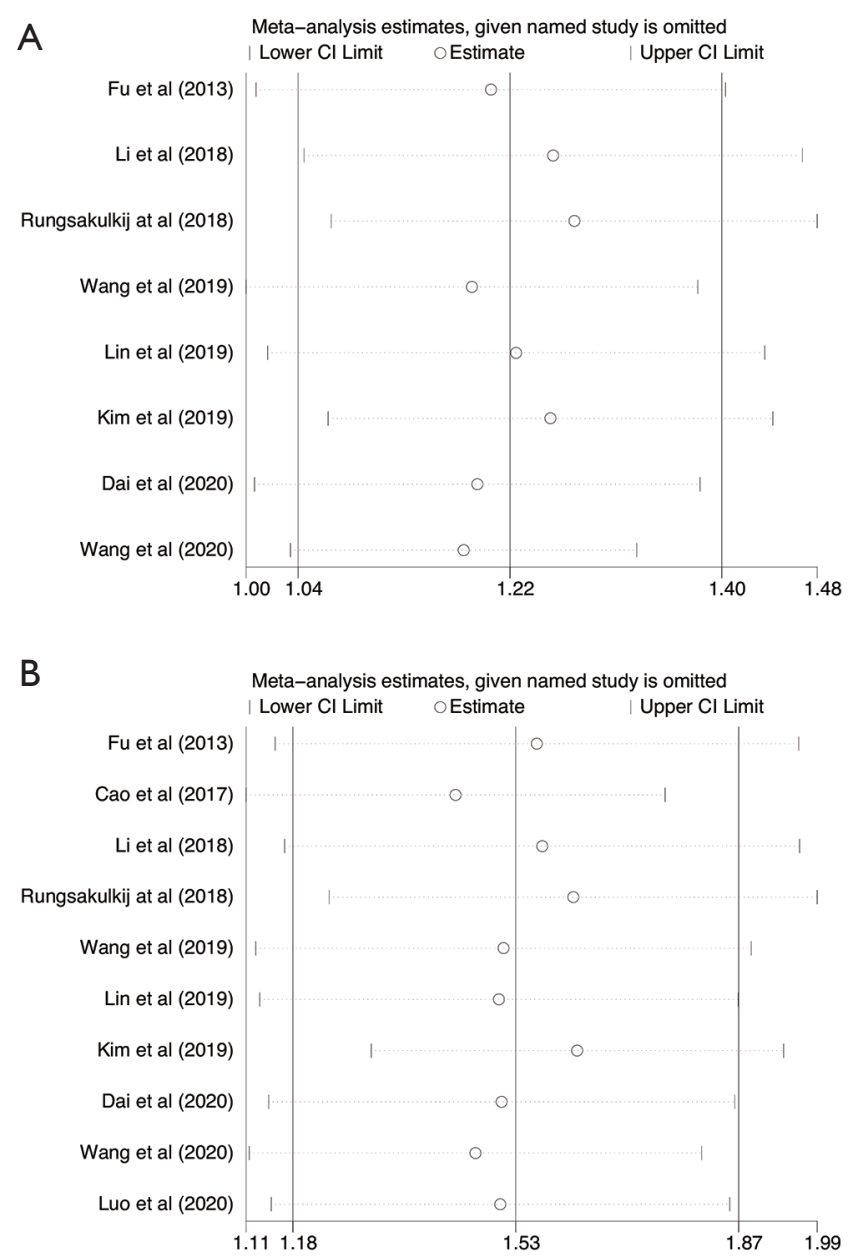

Figure 3 Results of the leave-one-out method for the impact of NLR on outcomes. (A) RFS. (B) OS. NLR, neutrophil to lymphocyte ratio; RFS, relapse-free survival; OS, overall survival.

types have different immune regulatory functions depending on the conditions, HBV infection status, and tumor stage. The CD8+ cell population represents active HBV clearance and anti-HCC tumor progression (33-37). The CD4+ and immune-suppressive immune cells such as myeloid-derived suppressor cells (MDSC) and Tregs often contribute to an immune-tolerant tumor microenvironment and poor clinical outcomes in HCC patients (38-42).

\section{Discussion}

NLR and PLR are often considered inflammatory markers and reflect cancer immune status. Inflammation is one of the hallmarks and driving factors of cancer (43). Tumor biology, including in HCC, is strongly regulated by multiple cytokines, such as interleukins (ILs), tumor necrosis factor (TNF), and interferons (INFs), which are secreted by a variety of inflammatory cells (44). Accordingly, the proportion of inflammatory cells that reflect cancer features are commonly considered as outcome predictors.

Inflammation is a critical factor involved in the pathogenesis of HCC. Numerous studies have reported the potential prognostic value of NLR and PLR in patients with postoperative HCC including HBV-related HCC. However, due to inconsistent results reported in those studies, it is still necessary to reassess their predictive role in these patients, especially those with HBV infection. In the present study, a pooled analysis was performed based on 11 eligible studies involving 5,083 HBV-infected HCC participants with initially resectable tumors. It was confirmed that both increased NLR and PLR significantly predicted poor RFS and OS without obvious publication biases. This result indicated that the recurrence and mortality risk of HBVrelated HCC patients who undergo tumor resection can be estimated by NLR and PLR levels. Our results are consistent with the findings of a previous meta-analysis indicating that NLR and PLR are reliable prognostic markers in HCC patients who have received multiple treatments, including transarterial chemoembolization, RFA, liver transplantation, and systemic therapies (45).

Several lines of evidence support the concept that HBV infection-related inflammatory and immune indexes are tightly associated with HCC tumor progression, recurrence, and relapse. It has been reported that HBV-DNA and hepatitis B surface antigen (HBsAg) levels are independent factors for predicting cancer recurrence in HCC patients who undergo hepatectomy (46). Moreover, Wang et al. found that regulating the systemic inflammation status could overcome postoperative recurrence in patients with HBV-related HCC (47). Therefore, anti-HBV therapy has been recommended as an effective treatment option for preventing HBV-related HCC recurrence (48). However, one retrospective study found that NLR and PLR partially reflected HBV-DNA and HBsAg levels in patients with chronic HBV infection-related diseases; this finding indicates that NLR and PLR could reflect HBV-induced chronic inflammation (49).

Neutrophils, as common inflammatory cells, play an important role in the tumor microenvironment and regulate the processes involved in immune escape, angiogenesis, and metastasis, by secreting multiple cytokines, including IL8, TNF- $\alpha$, vascular epidermal growth factor (VEGF), and matrix metalloproteinase 9 (MMP 9) (50,51). Peripheral 

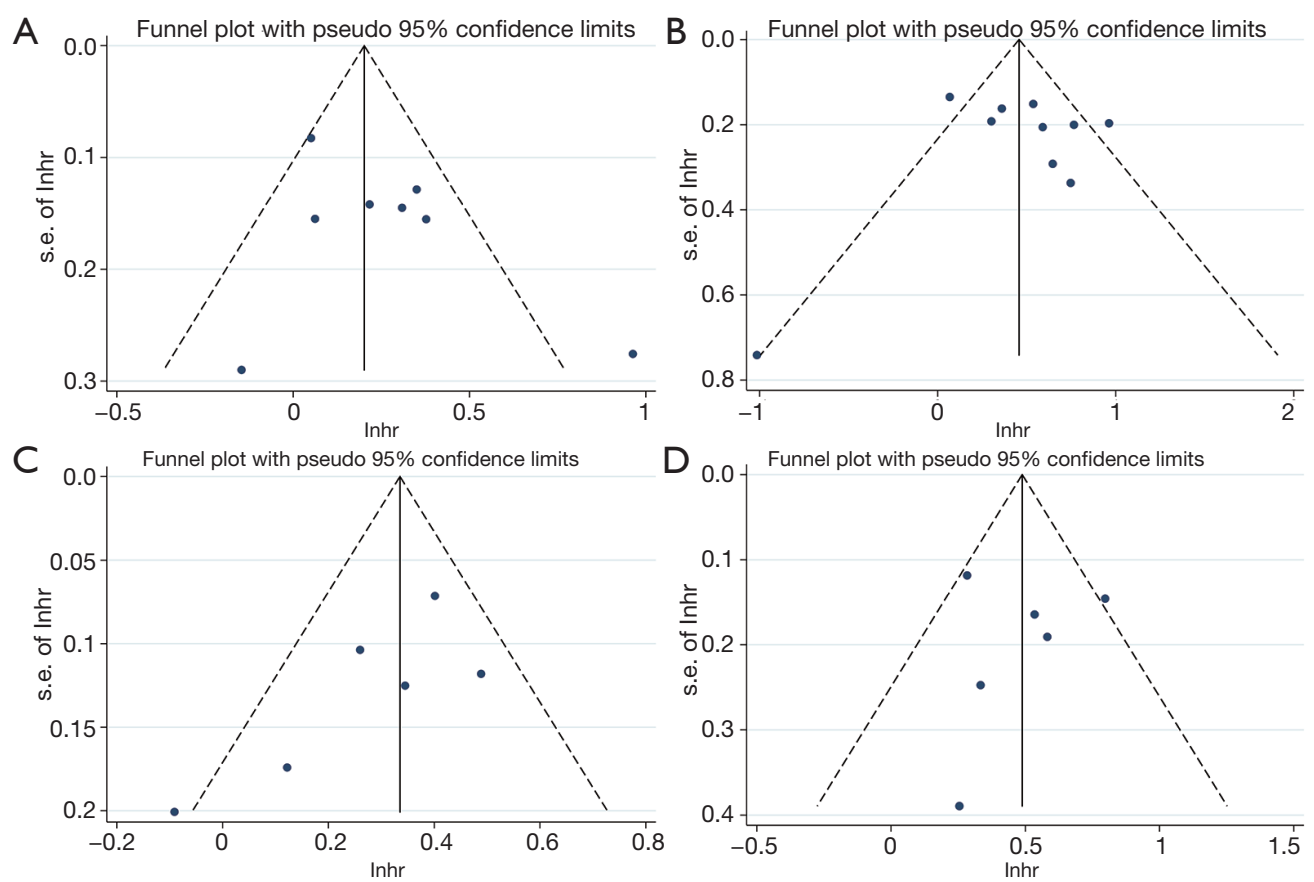

Figure 4 Funnel plots for assessment of publication bias. (A) RFS for NLR. (B) OS for NLR. (C) RFS for PLR. (D) OS for PLR. RFS, relapse-free survival; NLR, neutrophil to lymphocyte ratio; OS, overall survival; PLR, platelet to lymphocyte ratio.

Table 5 Neutrophil, platelets and immune cells associated with HBV infection, liver injury and HCC progression

\begin{tabular}{|c|c|c|}
\hline Cellular type & Immune regulatory functions & Study/Reference \\
\hline Neutrophil & $\begin{array}{l}\text { Promoting HCC progression by recruiting macrophages and Tregs infiltration. Inducing } \\
\text { resistance to sorafenib and angiogenesis via secreting multiple inflammatory cytokines }\end{array}$ & in vivo and in vitro (25) \\
\hline \multirow[t]{5}{*}{ Platelets } & $\begin{array}{l}\text { Promoting the accumulation of function-inefficient virus-specific CD8+ T-cells. Mediating liver } \\
\text { injury in status of chronic HBV infection. }\end{array}$ & in vitro (26) \\
\hline & Aggravating virus-induced immunopathology liver injury via deriving serotonin. & in vivo (27) \\
\hline & Directly binding to tumor cells and lead immune-escape and promoting cancer progression & in vivo (28) \\
\hline & Promoting HCC cells survival by cross-talk and secreting serotonin & in vivo and in vitro (29) \\
\hline & Stimulating HCC cell proliferation byvia IGF-1, HGF, TGF-beta, VEGF, PDGF-beta & In vivo and in vitro (30-32) \\
\hline \multirow{2}{*}{ CD8 } & HBV-specific CD8 T cells responses to immunotherapy for HCC patients & In vivo $(35,36)$ \\
\hline & Blocking HCC tumor progression & in vivo (37) \\
\hline \multirow[t]{2}{*}{ CD4 } & Indicated a poor survival in HCC patients & Cohort studies $(38,39)$ \\
\hline & HBV viral load regulates the PD-1 expression on CD4 T cells & Case-control study (40) \\
\hline MDSC & Involving in liver damage and reflecting systemic inflammation & Case-control study (41) \\
\hline Treg & Inhibiting the T cells functions in $\mathrm{HCC}$ microenvironment & Cohort study (42) \\
\hline
\end{tabular}


neutrophilia and increased neutrophil infiltration in the HCC tumor mass were found to be independent predictors of poor prognosis $(52,53)$. Lymphocyte percentage decreases when NLR or PLR increases. Lymphocytes, especially T lymphocytes, have been associated with a better prognosis in patients with postoperative HCC $(54,55)$. It has also been revealed that tumor-infiltrating lymphocytes regulate IL-17 and INF- $\gamma$ expression, thus contributing to the inhibition of HCC progression (56).

Platelets are multifunctional cells and are involved in inflammation and a variety of cancer biology-related processes. Several cytokines such as insulin-like growth factor-1 (IGF-1), epidermal growth factor (EGF), hepatocyte growth factor (HGF), fibroblast growth factor (FGF), VEGF, and platelet-derived growth factor (PDGF) are secreted by platelets, which contribute to the promotion of angiogenesis, immune escape, and metastasis (57-59). According to retrospective studies in HCC patients, thrombocytosis is significantly associated with large tumor size, high $\alpha$-fetoprotein levels, early extrahepatic metastasis, and poor OS and RFS $(60,61)$. Similar results were reported by a recent meta-analysis (62).

In summary, high NLR and PLR may reflect immune abnormalities in the cancer microenvironment, which have a negative influence on the outcomes of cancer patients. In this meta-analysis, we confirmed the prognostic value of these markers in HBV-related HCC patients who underwent hepatectomy.

Nevertheless, there were several limitations of this work that should be considered while interpreting these findings and applying these results in clinical practice: firstly, the cutoff values for NLR and PLR ranged widely among the studies, and further studies are needed to establish standard and optimal values for clinic practice. Secondly, most included studies were conducted in China, which may restrict the application of the findings to other areas. Thirdly, the HRs and their 95\% CIs were extracted from univariable analysis in several studies, which might have led to an overestimation of the prognostic value of these markers. Lastly, all enrolled studies were conducted retrospectively; therefore, it is necessary to perform largesample, well-designed studies to acquire high-quality evidence.

\section{Acknowledgments}

We thank professor J. Jones and J. Gray for polishing this manuscript.
Funding: Natural Science Foundation of Jiangsu Province (NO. BK20200275).

\section{Footnote}

Reporting Checklist: The authors have completed the PRISMA reporting checklist. Available at http://dx.doi. org/10.21037/tcr-20-3125

Peer Review File: Available at http://dx.doi.org/10.21037/tcr20-3125

Conflicts of Interest: All authors have completed the ICMJE uniform disclosure form (available at http://dx.doi. org/10.21037/tcr-20-3125). The authors have no conflicts of interest to declare.

Ethical Statement: The authors are accountable for all aspects of the work in ensuring that questions related to the accuracy or integrity of any part of the work are appropriately investigated and resolved.

Open Access Statement: This is an Open Access article distributed in accordance with the Creative Commons Attribution-NonCommercial-NoDerivs 4.0 International License (CC BY-NC-ND 4.0), which permits the noncommercial replication and distribution of the article with the strict proviso that no changes or edits are made and the original work is properly cited (including links to both the formal publication through the relevant DOI and the license). See: https://creativecommons.org/licenses/by-nc-nd/4.0/.

\section{References}

1. Bray F, Ferlay J, Soerjomataram I, et al. Global cancer statistics 2018: GLOBOCAN estimates of incidence and mortality worldwide for 36 cancers in 185 countries. CA Cancer J Clin 2018;68:394-424.

2. Bruix J, Takayama T, Mazzaferro V, et al. Adjuvant sorafenib for hepatocellular carcinoma after resection or ablation (STORM): a phase 3, randomised, double-blind, placebo-controlled trial. Lancet Oncol 2015;16:1344-54.

3. Ishizawa T, Hasegawa K, Aoki T, et al. Neither multiple tumors nor portal hypertension are surgical contraindications for hepatocellular carcinoma. Gastroenterology 2008;134:1908-16.

4. de Martel C, Georges D, Bray F, et al. Global burden of cancer attributable to infections in 2018: a worldwide 
incidence analysis. Lancet Glob Health 2020;8:e180-90.

5. Chan SL, Wong VW, Qin S, et al. Infection and Cancer: The Case of Hepatitis B. J Clin Oncol 2016;34:83-90.

6. Li ZL, Yan WT, Zhang J, et al. Identification of Actual 10-Year Survival After Hepatectomy of HBV-Related Hepatocellular Carcinoma: a Multicenter Study. J Gastrointest Surg 2019;23:288-96.

7. Zhang W, Wang R, Ma W, et al. Systemic immuneinflammation index predicts prognosis of bladder cancer patients after radical cystectomy. Ann Transl Med 2019;7:431.

8. Ishizuka M, Oyama Y, Abe A, et al. Combination of platelet count and neutrophil to lymphocyte ratio is a useful predictor of postoperative survival in patients undergoing surgery for gastric cancer. J Surg Oncol 2014;110:935-41.

9. Guo W, Lu X, Liu Q, et al. Prognostic value of neutrophilto-lymphocyte ratio and platelet-to-lymphocyte ratio for breast cancer patients: An updated meta-analysis of 17079 individuals. Cancer Med 2019;8:4135-48.

10. Li H, Zhao Y, Zheng F. Prognostic significance of elevated preoperative neutrophil-to-lymphocyte ratio for patients with colorectal cancer undergoing curative surgery: A meta-analysis. Medicine (Baltimore) 2019;98:e14126.

11. Chen K, Zhan MX, Hu BS, et al. Combination of the neutrophil to lymphocyte ratio and the platelet to lymphocyte ratio as a useful predictor for recurrence following radiofrequency ablation of hepatocellular carcinoma. Oncol Lett 2018;15:315-23.

12. Fu SJ, Shen SL, Li SQ, et al. Prognostic value of preoperative peripheral neutrophil-to-lymphocyte ratio in patients with $\mathrm{HBV}$-associated hepatocellular carcinoma after radical hepatectomy. Med Oncol 2013;30:721.

13. Cao Y, Jiang Z, Wang S, et al. Prediction of long-term survival rates in patients undergoing curative resection for solitary hepatocellular carcinoma. Oncol Lett 2018;15:2574-82.

14. Li C, Zhang XY, Peng W, et al. Preoperative albuminbilirubin grade plus platelet-to-lymphocyte ratio predict the outcomes of patients with BCLC stage A hepatocellular carcinoma after liver resection. Medicine (Baltimore) 2018;97:e11599.

15. Rungsakulkij N, Suragul W, Mingphruedhi S, et al. Prognostic factors in patients with $\mathrm{HBV}$-related hepatocellular carcinoma following hepatic resection. Infect Agent Cancer 2018;13:20.

16. Lo CK, Mertz D, Loeb M. Newcastle-Ottawa Scale: comparing reviewers' to authors' assessments. BMC Med
Res Methodol 2014;14:45.

17. Wang Y, Sun K, Shen J, et al. Novel Prognostic Nomograms Based on Inflammation-Related Markers for Patients with Hepatocellular Carcinoma Underwent Hepatectomy. Cancer Res Treat 2019;51:1464-78.

18. Lin ZX, Ruan DY, Jia CC, et al. Controlling nutritional status (CONUT) score-based nomogram to predict overall survival of patients with $\mathrm{HBV}$-associated hepatocellular carcinoma after curative hepatectomy. Clin Transl Oncol 2020;22:370-80.

19. Kim JM, Kwon CHD, Joh JW, et al. Nomograms in Hepatectomy Patients with Hepatitis B VirusRelated Hepatocellular Carcinoma. J Gastrointest Surg 2019;23:1559-67.

20. Dai T, Deng M, Ye L, et al. Prognostic value of combined preoperative gamma-glutamyl transpeptidase to platelet ratio and fibrinogen in patients with $\mathrm{HBV}$-related hepatocellular carcinoma after hepatectomy. Am J Transl Res 2020;12:2984-97.

21. Wang XH, Liao B, Hu WJ, et al. Novel Models Predict Postsurgical Recurrence and Overall Survival for Patients with Hepatitis B Virus-Related Solitary Hepatocellular Carcinoma $\leq 10 \mathrm{~cm}$ and Without Portal Venous Tumor Thrombus. Oncologist 2020;25:e1552-61.

22. Yang Y, Wang MC, Tian T, et al. A High Preoperative Platelet-Lymphocyte Ratio Is a Negative Predictor of Survival After Liver Resection for Hepatitis B VirusRelated Hepatocellular Carcinoma: A Retrospective Study. Front Oncol 2020;10:576205.

23. Luo D, Li H, Yu H, et al. Predictive value of preoperative and postoperative peripheral lymphocyte difference in hepatitis B virus-related hepatocellular cancer patients: Based on the analysis of dynamic nomogram. J Surg Oncol 2020;122:1553-68.

24. Li YW, Qiu SJ, Fan J, et al. Intratumoral neutrophils: a poor prognostic factor for hepatocellular carcinoma following resection. J Hepatol 2011;54:497-505.

25. Zhou SL, Zhou ZJ, Hu ZQ, et al. Tumor-Associated Neutrophils Recruit Macrophages and T-Regulatory Cells to Promote Progression of Hepatocellular Carcinoma and Resistance to Sorafenib. Gastroenterology 2016;150:164658.e17.

26. Iannacone $M$, Sitia $G$, Isogawa $M$, et al. Platelets mediate cytotoxic T lymphocyte-induced liver damage. Nat Med 2005;11:1167-9.

27. Lang PA, Contaldo C, Georgiev P, et al. Aggravation of viral hepatitis by platelet-derived serotonin. Nat Med 2008;14:756-61. 
28. Zhuang M, Xin G, Wei Z, et al. Dihydrodiosgenin inhibits endothelial cell-derived factor VIII and platelet-mediated hepatocellular carcinoma metastasis. Cancer Manag Res 2019;11:4871-82.

29. Soll C, Jang JH, Riener MO, et al. Serotonin promotes tumor growth in human hepatocellular cancer. Hepatology 2010;51:1244-54.

30. Matsuo R, Ohkohchi N, Murata S, et al. Platelets Strongly Induce Hepatocyte Proliferation with IGF-1 and HGF In Vitro. J Surg Res 2008;145:279-86.

31. He AD, Xie W, Song W, et al. Platelet releasates promote the proliferation of hepatocellular carcinoma cells by suppressing the expression of KLF6. Sci Rep 2017;7:3989.

32. Hoshi R, Murata S, Matsuo R, et al. Freeze-dried platelets promote hepatocyte proliferation in mice. Cryobiology 2007;55:255-60.

33. Maini MK, Boni C, Ogg GS, et al. Direct ex vivo analysis of hepatitis B virus-specific CD8(+) T cells associated with the control of infection. Gastroenterology 1999;117:1386-96.

34. Rehermann B, Ferrari C, Pasquinelli C, et al. The hepatitis $\mathrm{B}$ virus persists for decades after patients' recovery from acute viral hepatitis despite active maintenance of a cytotoxic T-lymphocyte response. Nat Med 1996;2:1104-8.

35. Maier H, Isogawa M, Freeman GJ, et al. PD-1:PD-L1 interactions contribute to the functional suppression of virus-specific CD8+ T lymphocytes in the liver. J Immunol 2007; 178:2714-20.

36. Probst HC, McCoy K, Okazaki T, et al. Resting dendritic cells induce peripheral CD8+ T cell tolerance through PD-1 and CTLA-4. Nat Immunol 2005;6:280-6.

37. Zong L, Peng H, Sun C, et al. Breakdown of adaptive immunotolerance induces hepatocellular carcinoma in HBsAg-tg mice. Nat Commun 2019;10:221.

38. Jia Y, Zeng Z, Li Y, et al. Impaired function of CD4+ T follicular helper ( $\mathrm{Tfh}$ ) cells associated with hepatocellular carcinoma progression. PLoS One 2015;10:e0117458.

39. Fu J, Zhang Z, Zhou L, et al. Impairment of CD4+ cytotoxic $\mathrm{T}$ cells predicts poor survival and high recurrence rates in patients with hepatocellular carcinoma. Hepatology 2013;58:139-49.

40. Xu P, Chen YJ, Chen H, et al. The expression of programmed death-1 in circulating CD4+ and CD8+ T cells during hepatitis $B$ virus infection progression and its correlation with clinical baseline characteristics. Gut Liver 2014;8:186-95.

41. Li T, Zhang X, Lv Z, et al. Increased Expression of
Myeloid-Derived Suppressor Cells in Patients with HBVRelated Hepatocellular Carcinoma. Biomed Res Int 2020;2020:6527192.

42. Lin SZ, Chen KJ, Xu ZY, et al. Prediction of recurrence and survival in hepatocellular carcinoma based on two Cox models mainly determined by FoxP3 + regulatory $\mathrm{T}$ cells. Cancer Prev Res (Phila) 2013;6:594-602.

43. Bishayee A. The role of inflammation and liver cancer. Adv Exp Med Biol 2014;816:401-35.

44. Gun SY, Lee SWL, Sieow JL, et al. Targeting immune cells for cancer therapy. Redox Biol 2019;25:101174.

45. Zheng J, Cai J, Li H, et al. Neutrophil to Lymphocyte Ratio and Platelet to Lymphocyte Ratio as Prognostic Predictors for Hepatocellular Carcinoma Patients with Various Treatments: a Meta-Analysis and Systematic Review. Cell Physiol Biochem 2017;44:967-81.

46. Sohn W, Paik YH, Kim JM, et al. HBV DNA and HBsAg levels as risk predictors of early and late recurrence after curative resection of HBV-related hepatocellular carcinoma. Ann Surg Oncol 2014;21:2429-35.

47. Wang Q, Blank S, Fiel MI, et al. The Severity of Liver Fibrosis Influences the Prognostic Value of InflammationBased Scores in Hepatitis B-Associated Hepatocellular Carcinoma. Ann Surg Oncol 2015;22 Suppl 3:S1125-32.

48. Wong JS, Wong GL, Tsoi KK, et al. Meta-analysis: the efficacy of anti-viral therapy in prevention of recurrence after curative treatment of chronic hepatitis B-related hepatocellular carcinoma. Aliment Pharmacol Ther 2011;33:1104-12.

49. Zhao Z, Liu J, Wang J, et al. Platelet-to-lymphocyte ratio (PLR) and neutrophil-to-lymphocyte ratio (NLR) are associated with chronic hepatitis B virus (HBV) infection. Int Immunopharmacol 2017;51:1-8.

50. Coffelt SB, Wellenstein MD, de Visser KE. Neutrophils in cancer: neutral no more. Nat Rev Cancer 2016;16:431-46.

51. Giese MA, Hind LE, Huttenlocher A. Neutrophil plasticity in the tumor microenvironment. Blood 2019;133:2159-67.

52. Margetts J, Ogle LF, Chan SL, et al. Neutrophils: driving progression and poor prognosis in hepatocellular carcinoma? Br J Cancer 2018;118:248-57.

53. Zhou SL, Dai Z, Zhou ZJ, et al. Overexpression of CXCL5 mediates neutrophil infiltration and indicates poor prognosis for hepatocellular carcinoma. Hepatology 2012;56:2242-54.

54. Nakagawa S, Umezaki N, Yamao T, et al. Survival impact of lymphocyte infiltration into the tumor of hepatocellular carcinoma in hepatitis B virus-positive or non-B non-C 
patients who underwent curative resection. Hepatol Res 2018;48:E126-32.

55. Ding $W, X u X$, Qian $Y$, et al. Prognostic value of tumorinfiltrating lymphocytes in hepatocellular carcinoma: A meta-analysis. Medicine (Baltimore) 2018;97:e13301.

56. Wang WC, Zhang ZQ, Li PP, et al. Anti-tumor activity and mechanism of oligoclonal hepatocellular carcinoma tumor-infiltrating lymphocytes in vivo and in vitro. Cancer Biol Ther 2019;20:1187-94.

57. Bambace NM, Holmes CE. The platelet contribution to cancer progression. J Thromb Haemost 2011;9:237-49.

58. Senzel L, Gnatenko DV, Bahou WF. The platelet proteome. Curr Opin Hematol 2009;16:329-33.

Cite this article as: Zheng X, Ye B, Gou Y, Li Z, Chen C, Liao F, Liu X, Qin S. Neutrophil to lymphocyte and platelet to lymphocyte ratios as biomarkers to predict relapse and survival in posthepatectomy $\mathrm{HBV}$-related hepatocellular carcinoma: a meta-analysis and preliminary immune perspective. Transl Cancer Res 2021;10(3):1261-1272. doi: 10.21037/tcr-20-3125
59. Rachidi S, Metelli A, Riesenberg B, et al. Platelets subvert $T$ cell immunity against cancer via GARP-TGF $\beta$ axis. Sci Immunol 2017;2.

60. Scheiner B, Kirstein M, Popp S, et al. Association of Platelet Count and Mean Platelet Volume with Overall Survival in Patients with Cirrhosis and Unresectable Hepatocellular Carcinoma. Liver Cancer 2019;8:203-17.

61. Hwang SJ, Luo JC, Li CP, et al. Thrombocytosis: a paraneoplastic syndrome in patients with hepatocellular carcinoma. World J Gastroenterol 2004;10:2472-7.

62. Ma W, Zhang P, Qi J, et al. Prognostic value of platelet to lymphocyte ratio in hepatocellular carcinoma: a metaanalysis. Sci Rep 2016;6:35378. 